\title{
DILEMAS ÉTICOS Y PEDAGÓGICOS EN LA INDUSTRIA DE ALIMENTOS EN MÉXICO. PATRONES DE PROMOCIÓN Y FORMACIÓN DE HÁBITOS ALIMENTICIOS
}

\author{
ETHICAL AND PEDAGOGICAL DILEMMAS \\ IN THE MEXICAN FOOD INDUSTRY. \\ PROMOTION PATTERNS AND FORMATION \\ OF EATING HABITS
}

\author{
Gustavo Adolfo Esparza Urzúa ${ }^{1}$ \\ https:// orcid.org/0000-0002-9470-6519 \\ María del Carmen Gutiérrez Guerrero ${ }^{2}$ \\ https://orcid.org/0000-0001-7542-6274
}

Julieta Domínguez Soberanes ${ }^{3}$

Recibido: mayo 7, 2020 - Aceptado: mayo 31, 2020

\section{RESUMEN}

El objetivo del presente artículo es identificar los patrones pedagógicos que existen en los procesos de consumo de alimentos. El problema del que partimos considera dos aristas: primera, según los reportes de salud, nuestro país padece sobrepeso, obesidad y malnutrición; segundo, en lo que respecta a las industrias de alimentos, si bien operan bajo una lógica de negocios (mercadológica, directiva, administrativa, etcétera), las estrategias de promoción en su conjunto están diseñadas para formar conductas comunitarias, de tal modo que parte de los problemas de alimentación provienen de una intencionalidad sistemática.

1 Doctor en Ciencias Sociales y Humanidades, Departamento de Filosofía, Universidad Autónoma de Aguascalientes, México. Miembro del Sistema Nacional de Investigadores, nivel 1. Profesor investigador del Departamento de Humanidades, Universidad Panamericana, Aguascalientes, México.gaesparza@up.edu.mx

2 Doctora en Ciencias Biológicas, Universidad Autónoma de Aguascalientes en colaboración con la Universidad Autónoma de San Luis Potosí, México. Profesora investigadora en la Escuela de Negocios Gastronómicos, Universidad Panamericana, Aguascalientes, México.mcgutierrez@up.edu.mx

3 Doctora en Doctora en Ciencias Biológicas, Universidad Autónoma de Aguascalientes, México. Miembro del Sistema Nacional de Investigadores, nivel 1. Secretaria de investigación, Escuela de Negocios Gastronómicos, Universidad Panamericana, Aguascalientes, México. jdominguez@up.edu.mx 
A través de una investigación documental, logramos dos resultados: 1) una visibilización de los procesos de venta de alimentos como una pedagogía del consumidor, y 2) el diseño de algunas estrategias de intervención a largo plazo para incluir el concepto de «dignidad de la persona», como parte de las estrategias directivas de los negocios gastronómicos.

Palabras clave: nutrición, alimentación, industria de alimentos, dignidad de la persona, antropología del consumidor, pedagogía del consumidor.

\begin{abstract}
The purpose of this article is to identify the pedagogical patterns within food consumption processes. The problem considers two aspects; first, according to health reports, our country suffers from overweight, obesity and malnutrition. Second, with regard to the food industries, although they operate under a business logic (marketing, management, administrative, etc.), the promotion strategies as a whole are designed to form community behaviors, in such a way that part of the feeding problems come from a systematic intentionality. Therefore, through a documentary research we achieved two results: 1) visibility of the food sale processes as a consumer pedagogy and 2) the design of some long-term intervention strategies to include the concept of «dignity of the person» as part of the management strategies of gastronomic businesses.
\end{abstract}

Keywords: Nutrition, Food, Food industry, Dignity of the person, Consumer anthropology, Consumer pedagogy.

\title{
1. INTRODUCCIÓN. LÍMITES Y LIMITACIONES DE LA PERSONA COMO CONSUMIDOR
}

El objetivo del presente trabajo es presentar parte de los resultados derivados de la línea de investigación Cultura alimentaria y hospitalidad, y de las clases de pregrado en la Escuela de Dirección de Negocios Gastronómicos (DNG), así como en la Maestría en Desarrollo e Innovación de Alimentos (MDIA). Uno de los resultados más importantes que anima la redacción de este estudio es un sondeo realizado a los alumnos inscritos, por parte de la coordinación administrativa y en las sesiones (por ahora sincrónicas) por parte de los docentes; advertimos, en esta aproximación, diversas concepciones epistemológicas básicas con las cuales se construyen los negocios gastronómicos. A continuación clasificamos algunos de estos hallazgos para clarificar el problema pedagógico al que deseamos referirnos:

- Proceso de inscripción de los alumnos: el interés de los alumnos por cursar esta maestría ha ido en aumento a lo largo de la historia del posgrado. Las expectativas generales de los alumnos son: «Interés por encontrar mejores estrategias de venta, distribución y promoción del producto gastronómico en los mercados actuales y potenciales de distribución»; «Espero encontrar mejores estrategias para el crecimiento de mi negocio y, de acuerdo con lo que se ofrece, desarrollar procesos que permitan atender mejor el recurso humano»; «Encontrar un balance entre el negocio gastronómico y la dignidad de la persona humana, en cuanto consumidor de mis productos». 
- Proceso de formación académica general: los alumnos manifiestan su agrado general con respecto del desarrollo del programa. La formación de habilidades es evidente conforme se acreditan los módulos, pero también, y debido a la misión y visión de la Universidad Panamericana (UP), las clases se encuentran imbuidas por una pedagogía centrada en la persona (Llergo y Nicolás, 2018). Lo relevante de este aspecto es que la formación técnico científica de las diversas sesiones -a pesar de no pretender un contenido antropológico-, orienta los fundamentos de sus contenidos hacia formatos de negocios cuyo desarrollo último favorece las necesidades de consumo gastronómico de la persona.

- Proceso de formación académica institucional: siendo el pilar central de la pedagogía la formación de la persona, se ha dispuesto que las materias institucionales de la UP operen bajo los modelos referentes de la Paideia y el Humanitas cristiano (Llergo y Nicolás, 2018). El efecto inmediato de esta orientación ha permitido que los alumnos manifiesten explícitamente una reconsideración de sus modelos de negocios, con el fin de incorporar en su administración y gestión a la "persona». Lo relevante de este resultado se desglosa en dos preguntas que regularmente expresan los alumnos: ¿cómo fundamentar la estructura del negocio gastronómico a partir de la persona, sin atentar contra la rentabilidad?, ¿cuál es el modelo de negocio gastronómico con que se puede dignificar a los consumidores?

- Revisión del estado de la cuestión en lo que respecta a los procesos de consumo y su relación con los negocios gastronómicos: parte de los resultados hallados en la indagación de los referentes teóricos que fundamenten nuestra línea de investigación, constata un patrón social que nos ha llamado la atención. Como se verá más adelante, existe un interés generalizado, de parte de las industrias gastronómicas, por privilegiar el consumo de sus productos, pero apoyándose en procedimientos de promoción de acciones que beneficien la compra-venta del producto, sin que necesariamente importe los efectos que causan en la salud del consumidor final.

Aclaramos así el problema al que deseamos referirnos: en México existen patrones de alimentación que históricamente han sido fomentados por industrias cuyo interés es la promoción del consumo; sin embargo, en la construcción de su modelo de negocio han descuidado el valor nutricional y el valor antropológico (Moss, 2013; López-Ojeda et al., 2017, 93-97). Dicha promoción, si bien se ha impulsado a través de estrategias mercadológicas (comerciales, publicidad, estructuración de clúster de consumidores, etcétera), apunta a lo que Enrique Moreno y de los Arcos (1993) llama "proceso pedagógico asistemático», convirtiéndose en un proceso de formación de consumo de ciertos alimentos predeterminados por algunas industrias transnacionales, a partir de patrones de promoción de alimentos que se traducen en sistemas de creación de conductas comunitarias. En este sentido, advertimos algo más que una estrategia publicitaria de creación de necesidades: enfrentamos una situación social en la que, debido a la gran cantidad de recursos, puede hablarse de un sistema de regulación de hábitos comunitarios, que se traduce en un proceso pedagógico social.

La principal inquietud detectada en las distintas materias, impartidas al interior de la Escuela de Dirección de Negocios Gastronómicos -así como en el sondeo realizado con los alumnos de distintas generaciones-, advierte una tendencia social por impulsar el consumo de algunos alimentos a partir de principios mercadológicos, de negocios, de comunicación, los cuales, en su conjunto, orientan los hábitos personales. Para nosotros, por ende, es importante estructurar y promover la visibilización de los procesos pedagógicos que subyacen en la actual industria de alimentos en México. 
Reflexionar sobre los cauces conductuales que prevalecen dentro del mercado de alimentos, es el primer paso para crear estrategias de reconducción de prácticas y hábitos de consumo de la persona, pero al mismo tiempo deben integrarse armónicamente tres cuestiones: dignificación de la persona como concepto clave de la estructura del negocio; rentabilidad de modelos de negocios gastronómicos, y legalidad de los procesos industriales y de promoción de producto. Finalizamos con algunas sugerencias que actualmente se proponen en el programa, mencionando algunas líneas del proyecto de intervención que hoy se encuentra en desarrollo.

\section{DIAGNÓSTICO DEL PROBLEMA: PATRONES DE CONSUMO DE ALIMENTOS EN MÉXICO}

En México, la mala alimentación constituye un problema ya que, según los datos de la última Encuesta Nacional de Salud y Nutrición (ENSAUT), la prevalencia nacional de sobrepeso u obesidad supera el promedio mundial (ENSAUT 2019). Las cifras relacionadas con la salud nutricional, tienen encendidos los focos rojos desde hace tiempo: la malnutrición abarca la desnutrición (emaciación, retraso del crecimiento e insuficiencia ponderal), los desequilibrios de vitaminas o minerales, el sobrepeso, la obesidad, y las enfermedades no transmisibles relacionadas con la alimentación, entre ellas la diabetes, las cardiopatías, los accidentes cerebrovasculares y el cáncer (Denham et al. 2020; ENSAUT, 2019). Para contextualizar, los datos recabados por la ENSAUT del INEGI -levantamiento del 30 de julio de 2018 al 28 de junio de 2019- señalan que 57\% de los hogares mexicanos se encuentran en inseguridad alimentaria: de esta, $15.5 \%$ es severa; $24.5 \%$ moderada y $60 \%$ leve.

La inseguridad alimentaria se define como la disponibilidad limitada o incierta de alimentos nutricionalmente adecuados e inocuos; o la capacidad limitada e incierta de adquirir alimentos adecuados en formas socialmente aceptables (Ibarrola-Rivas et al. 2017). Con el fin de que estos problemas relacionados con la mala alimentación y con la desinformación nutricional puedan mitigarse, y que la salud de las personas tienda hacia el bienestar, consideramos necesario desarrollar estrategias educativas que difundan la información nutricional relevante provocando, en un futuro cercano, un impacto positivo en la salud pública de nuestro país.

La literatura crítica identifica diversas causas de esta situación, algunas de ellas son: las vinculadas con hábitos alimenticios relacionados con el nivel sociodemográfico y económico de los consumidores (Gaona-Pineda et al., 2018; Gil-Toro, 2019; ENSAUT, 2019); las concernientes a los efectos de la publicidad (Marrón-Ponce, 2021; Márquez, 2021); las derivadas de una falta de regulación jurídica estricta que delimite los efectos publicitarios en el consumidor (Márquez, 2021); las relacionadas con la información nutricional disponible y su claridad, por ejemplo en el etiquetado de alimentos (Anastasiou et al., 2019; Sunstein, 2021) y, finalmente, las estrategias comerciales y de distribución de alimentos, promovidas por la propia industria de alimentos (Taddei-Bringas, 2006; Ayala-Ramírez; Castillón-Girón, 2014).

Respecto del tipo de alimentación y su relación con el nivel sociodemográfico y económico, dentro de la ENSAUT (2019) se presentan esfuerzos por garantizar el consumo de alimentos a través del impulso de distintos programas; si bien existe una proporción de ayuda según las necesidades de la región del país (los hogares reciben apoyo: sur, 50\%; 
centro, 32\%; norte, $22.4 \%$ ), se aclara que aún existen sectores vulnerables desprotegidos, tales como: cocinas del DIF, albergues indígenas, comedores de la Secretaría de Desarrollo Social, y los apoyos a Organizaciones No Gubernamentales, apenas con el 1\% (ENSAUT, 2019).

Estudios previos a esta fecha, ya indicaban que a mayor nivel socioeconómico, existe un consumo de alimentos recomendables (Galván-Portillo et al., 2018; Jansen et al., 2020). Luego de la aparición del SARS-CoV-2, en el año 2020, los estudios muestran que los hábitos alimenticios arraigados en las distintas zonas del país, así como en su nivel económico, son un factor de riesgo a tomar en cuenta (Vique-Sánchez, 2021).

La publicidad ha demostrado ser un factor de incidencia en los productos de consumo en lo general, pero especialmente en la edad infantil. A mayor tiempo de exposición de publicidad, mayores las probabilidades de consumo no saludable (Pérez-Salgado, 2010; Castronouvo, 2016). Otros, en cambio, muestran que existen estrategias diferenciadas para favorecer el consumo de alimentos no saludables en zonas de niveles socioeconómicos bajos y, en cambio, fomentar un consumo saludable en niveles socioeconómicos altos (Vique-Sánchez, 2021). En contraparte, se tiene evidencia de los esfuerzos específicos por parte del gobierno mexicano, para garantizar que la publicidad promueva hábitos comunes entre los consumidores (Suárez Solana, 2016). Sin embargo, no se han logrado los efectos esperados: algunos estudios muestran que una buena parte del problema se debe a la falta de información con la cual los consumidores interpretan la información nutrimental y el etiquetado de los alimentos (Tolentino-Mayo et al., 2021).

Respecto de las estrategias mercadológicas de promoción de los alimentos, por parte de las industrias, llama la atención la diferenciación que se encuentra en los estudios sobre ese tema. Por un lado, es claro que las industrias mayoristas o de alto nivel de influencia comercial enfocan sus actividades comerciales al fortalecimiento de su posición como grupo de influencia, favoreciendo estrategias que les permitan mantener las redes de distribución y la preferencia comercial; algunos de los recursos identificados por Taddei-Bringas (2006), reflejan que se trata de acciones encaminadas a garantizar la presencia de la firma y no así de la mejora de los nutrimentos, entre los ejemplos tenemos: compra, venta de acciones y/o alianzas comerciales con otras empresas que garanticen el control de ventas de un cierto producto de preferencia, también el acaparamiento del producto primario, el control de las rutas de distribución, así como de las preferencias entre los consumidores al orientar el tipo de alimentación que se consume (esto por medio del tipo de productos que se distribuyen y que son identificados comunitariamente como productos líderes), lo que hace pensar si la calidad nutrimental del producto forma parte de sus estrategias de posicionamiento.

En esta línea son interesantes los resultados alcanzados por Lorena Carrete, Pilar Arroyo y Edgar Centeno (2018), quienes exponen el problema al que se enfrentan las industrias de alimentos en lo que respecta a las estrategias publicitarias de sus productos. De acuerdo con las autoras, el gran dilema es que, una vez posicionado un producto considerado como «no saludable», la reconversión hacia la venta de productos «saludables» representa una pérdida de credibilidad del consumidor en torno a los que dicha marca ofrece; ello no solo hace que exista una renuencia hacia el nuevo producto promocionado, sino que se dude del ofertado previamente. Para efectos prácticos, esto se traduce en un dilema que implica evaluar el costo de impacto, una vez que una empresa quiere impulsar un cierto tipo de alimento con mayor valor nutricional. Esto es importante porque, dados estos resultados, parece que las empresas deben optar por una presencia comercial que garantice el cumplimiento de sus objetivos, o bien costear la reconducción para alcanzar las preferencias por parte de los consumidores. 
Con esta aproximación desde el estado de la cuestión, se señalan dos temas:

1. La evidencia empírica manifiesta que los hábitos alimenticios adquiridos no corresponden únicamente a quienes consumen un cierto producto, sino que también participan mecanismos sociales: publicidad, distribución del producto y estrategias de regulación gubernamental.

2. Con independencia de los objetivos de las industrias -sea, por ejemplo, el del desarrollo de estrategias puramente comerciales-, la traducción de sus acciones se manifiesta en hábitos, no necesariamente saludables, promovidos entre los consumidores. Se puede argumentar que es responsabilidad exclusiva de quien acepta ciertos alimentos esa falta de un orden alimenticio pero, como ya se ha mostrado, los estudios manifiestan que existe un impulso intencional para favorecer ciertos productos, lo cual refleja el papel esencial que juegan las industrias en el desarrollo de la salud pública en México (Hernández-F. et al., 2021).

De acuerdo con esto, si bien existen estudios diagnósticos y recomendaciones para cada problema detectado, es necesario considerar como solución complementaria a todos estos esfuerzos, el fortalecimiento de estrategias pedagógicas que incidan en la formación de hábitos alimenticios y de conceptos saludables de consumo.

Aunque existen enfoques pedagógicos diversos -relacionados con las modificaciones de conducta-, la perspectiva sociocrítica de Vigotsky (1986) muestra, en particular, que se logran nuevos patrones de comportamiento en la medida en que exista una resignificación de las acciones que comúnmente se realizan, una vez expuesto un razonamiento argumentado, acorde a la edad evolutiva del sujeto en cuestión. Por ende, consideramos que la implementación de nuevas estrategias en la formación de hábitos alimenticios, debe iniciar diagnosticando algunos de los conceptos básicos que predominan en la elección de un alimento, para luego ofrecer una argumentación diversificada en estrategias tales como: exposiciones, debates, diálogos dirigidos, plenarias, promoción de exposiciones de parte de los propios alumnos, entre otros. La finalidad es favorecer la apropiación de los argumentos centrales a tomarse en cuenta para la significación personal de las conductas deseadas.

En el siguiente apartado presentaremos esfuerzos desarrollados por el gobierno mexicano para mostrar que el trasfondo de la estrategia de la industria de alimentos se encamina a fomentar hábitos específicos de consumo, y que no solo se trata de decisiones individuales de los consumidores al elegir los productos.

\section{ESTRATEGIAS PEDAGÓGICAS DE PROMOCIÓN DE CONSUMO DE ALIMENTOS EN MÉXICO}

Debido a que nuestro objetivo se encamina a mostrar los patrones y procesos a través de los cuales los consumidores eligen ciertos alimentos, no desarrollaremos una definición puntual de lo que se entiende por pedagogía. Partimos de los siguientes supuestos conceptuales:

1. La pedagogía es la ciencia de la educación (Larroyo, 1969). Al asumir esta delimitación, aceptamos los presupuestos filosóficos -específicamente neokantianos- en los que Larroyo funda su visión pedagógica; ello implica que entendemos la distinción entre formación (Bildung) y educación (Erziehung). Por la primera se entiende un proceso de orientación integral de facultades humanas, y por la segunda, uno de crecimiento y adaptación del individuo a 
su entorno (cfr. Esparza, 2019). Precisamente este segundo aspecto justifica la terminología «pedagogía del consumo» por parte de las industrias, aun cuando sus objetivos no estén centrados en dignificar a la persona.

2. Algún proceso pedagógico no es sistemático, aunque necesariamente es técnico y artístico (Ruiz, 1904; Moreno, 1993; Esparza, 2019). Esta es la tesis que desarrollaremos con más amplitud en lo que resta del artículo, aunque no la justificamos directamente. Según los autores consultados, la pedagogía -si bien es una ciencia y por ello posee un método científico desde el cual opera- es también un procedimiento artístico. Apuntamos únicamente que por método científico no entendemos un modo de comprender naturalizado (caracterizado por su afán estadístico-descriptivo), sino que ubicamos esta ciencia dentro del ámbito de las ciencias humanas, por lo que proponemos que el objetivo de la ciencia es la comprensión hermenéutica en el sentido filosófico del término (y no en el sentido naturalizado del término). Por el concepto de arte, reconocemos la acepción técnico creativa imbuida dentro del mundo griego, pues consideramos que el discernimiento de la selección de estrategias de fomento de nuevas habilidades puede entenderse como la tarea central de la actividad pedagógica (Junco, 2018; Esparza, 2021).

Partiendo de ambos aspectos, mostraremos algunas de las estrategias intencionales con las cuales se promueven ciertos alimentos en nuestro país. El equipo de Irving Márquez et al. (2020) estudia las regulaciones en materia de control publicitario, impuestas a las industrias alimentarias. Ellos parten del siguiente indicador: «[...] de 2 a 12 años pasa un promedio de 4 horas con 46 minutos diarios frente a la televisión; al comparar con información de 2016, su exposición aumentó 22 minutos diarios[ ...] se estima que por cada hora de televisión, el riesgo de obesidad incrementa en 12\%» (p. 93, énfasis añadido).

Esto significa que en México la población infantil no consume una dieta balanceada, en parte debido a la promoción publicitaria de la TV y Radio. Partiendo de este hecho, señalan que el objetivo de las leyes para la regulación de la publicidad en alimentos -aunque se ha propuesto garantizar el derecho a la salud de los consumidores-, debe ser más estricto para limitar algunas prácticas que de facto existen en nuestro país.

Por otro lado, Elsa B. Gaona-Pineda et al. (2018) manifiestan que, independientemente de las causas, es una realidad que en México existe «[...] una disminución del gasto per cápita en alimentos recomendables como frutas, verduras, lácteos y carnes, y en un mayor gasto en alimentos densos en energía (como cereales refinados y comida rápida) y bebidas azucaradas» (p. 273).

De acuerdo con el grupo de investigación, dicha variación en el gasto se debe centralmente a factores relacionados con el Nivel Socio Económico (NSE): «El NSE está asociado con el ingreso, por lo que, en NSE más altos, es posible que se presente mayor acceso a alimentos y mejor calidad de la dieta» (p. 273), lo que desafortunadamente se traduce en que la población con mayores necesidades económicas, consume alimentos menos saludables que aquella porción poblacional que cuenta con mayores recursos. Estos resultados ya habían sido detectados por el ENSANUT, en donde se indica que: «Los programas de ayuda alimentaria forman parte de las estrategias gubernamentales para atender a las necesidades de la población [...] con mayor frecuencia los grupos vulnerables» (p. 161). El problema, sin embargo, no se resuelve atendiendo a sectores específicos de la población, sino que se debe emprender una estrategia integral.

El grupo de Joaquín Marrón et al. (2020) plantea que una parte importante del problema de la alimentación en México, proviene del etiquetado del producto. La tesis es que comemos lo que se nos ofrece publicitariamente, aun cuando han existido diversas modificaciones a 
las leyes que norman esta cuestión (Irving Márquez et al., 2020). Según el estudio de Marrón y su equipo, existe una diferencia en la estrategia de ventas en las regiones de nivel alto y bajo. Este estudio recolectó un total de 7,567 alimentos, sectorizando la población su NSE quedando como sigue: 4,826 en el NSE alto; mientras que 2,741 de los sujetos de estudio pertenecían al NSE bajo. Los autores ofrecen tres resultados: 1) en ambos NSE se observó que los supermercados son el principal punto de venta para encontrar una oferta más variada de publicidad; 2) se identificó que los supermercados del NSE alto tienen mayor porcentaje de estrategias publicitarias que promueven el consumo de alimentos saludables (19.9\%), en comparación con los supermercados del NSE bajo (12.3\%) y, finalmente, 3):

[...] aquellos puntos de venta situados en áreas marginadas son más propensos a ofertar alimentos con un perfil nutricional menos saludable [en contraparte,] se observó que la proporción de [publicidad de alimentos] saludables fue mayor en los puntos de venta del NSE alto que en los de NSE bajo (Marrón et al., 2020, p. 85).

Estos datos muestran que por parte del gobierno federal se impulsan programas para la promoción, la distribución y el consumo de alimentos saludables, y que los puntos de venta y consumo regulares asumen patrones de publicidad diferenciados relacionados directamente con el NSE, lo que se traduce en una estrategia contrapuesta en lo que respecta a la promoción de conductas. Esto es importante porque la promoción de conductas saludables depende del sector económico al que se atiende. En este sentido, existen al menos dos componentes básicos para la formación de una conducta: a) una intencionalidad estratégica en la que se reconoce el perfil del sujeto (psicología) y la poblacional (sociología) de los consumidores, y b) un modo de proceder consistente con dichos indicadores, con el fin de impulsar una conducta predefinida (planeación estratégica).

Las aseveraciones anteriores no son novedosas ni pretenden desentrañar un patrón de conducta oculto. La propia literatura de los alimentos se ha esforzado por lograr estos principios; muestra de ello es el libro de Salt, Sugar, Fat (Moss, 2013) o los estudios relacionados con la modelación de intereses de consumo realizados por Moskowitz (1994). Subrayamos que en ningún caso consideramos que la metodología desarrollada por este último autor, por citar un ejemplo, deba asumirse como un esfuerzo por parte de la industria de alimentos por vulnerar la salud de la población. Únicamente apuntamos que existe una lógica de optimización del producto.

Ya desde hace años, la literatura crítica vinculada al consumo de alimentos, reconocía las posibilidades de modelar y de orientar los hábitos del consumidor. Hal MacFie y D. Thomson, en un libro clásico, Measurement of Food Preferences (1994), reconocen que la medición de preferencias es un proceso multifactorial cuya interrelación fortalece algunos hábitos o desestimula otros, dependiendo de los procedimientos de influencia que interaccionen y se regulen externamente mediante algunos recursos predefinidos.

Lo anterior es importante porque se reconoce un marco metodológico estructurado para el fomento de hábitos de consumo. En lo que respecta a la pedagogía, según se planteó al inicio de esta sección, se señaló que esta ciencia tenía como objeto formal la «educación», es decir, el desarrollo de conductas de adaptación del ser humano al medio circundante. La cuestión central en este aspecto, y en relación con lo planteado, es que el desarrollo de hábitos de consumo se enmarca dentro de un sistema de medición de preferencias que fácilmente regulan y orientan el interés poblacional por aceptar o rechazar cierto tipo de alimentos.

En México, de acuerdo con el ENSAUT (2019), no comemos saludablemente pues enfrentamos problemas de consumo y de obesidad. Si bien no puede afirmarse que exista un 
patrón de orientación de las conductas que deriven en estas condiciones de salud de los consumidores, es importante insistir que los estudios sobre la publicidad y el etiquetado que promueven las preferencias de consumo, ofrecen resultados similares.

Según Lizbeth Tolentino-Mayo et al., (2020), aunque existen esfuerzos sistemáticos por parte del gobierno federal para cambiar tanto las conductas como el tipo de alimentos que se consumen, es claro que la estrategia vinculada a lo que hoy se conoce como «etiquetado frontal de alimentos y bebidas industrializados» (modificación de la NOM-051-SCFI /SSA1-2010), resulta insuficiente:

[...] es importante reconocer que el etiquetado de advertencia no es la panacea para resolver la emergencia epidemiológica de la obesidad y las ECNT, pero forma parte de una serie de políticas multisectoriales que se necesitan para salvaguardar la salud pública (Tolentino-Mayo et al., 2020, p. 796).

A pesar del esfuerzo por mejorar la comprensión del tipo de alimento que se ofrece y de las campañas de información que existen para explicar las consecuencias -benéficas y perjudiciales- de los diversos tipos de alimentos, la literatura crítica ha sido unánime en insistir que la atención es multifactorial y debe proceder de un modo más sistemático, procurando atender factores diversos. A continuación se exponen algunas de las propuestas más importantes al respecto.

\section{ESTRATEGIAS PEDAGÓGICAS DE INTERVENCIÓN PARA LA MEJORA DEL CONSUMO DE ALIMENTOS}

Como se expuso al inicio del apartado anterior, la pedagogía es una ciencia cuyo objeto de estudio es la «educación», pero también la «formación» ${ }^{4}$. De acuerdo con esto, podemos señalar algunas cuestiones cruciales:

- La pedagogía no puede entenderse únicamente a través de un prisma didáctico, sino que debe asumir una visión sistemática o comunitaria. Buena parte de las tendencias actuales se encaminan a profundizar y estudiar los procesos de enseñanza aprendizaje dentro del aula, aunque también en diversos contextos sociales. Según los referentes clásicos, la pedagogía no es únicamente un modo de comprensión instrumental sino de procesos integrales (Comenio, 1991; Nout, 2017; Esparza, 2018).

- Los procesos de intervención pedagógica no se reducen a la formación de conductas en la persona «individual», sino en la comunidad de personas. Consideramos que si bien la pedagogía debe asumir a la persona como su centro (Llergo y Nicolás, 2018), es necesario reconocer el valor de la persona como agente que vive y se desarrolla en comunidad. No podemos considerar a la persona únicamente en su dimensión individual, desprendiéndola de sus implicaciones y relaciones con el resto de miembros de la comunidad (Calabrese, 2018; Junco, 2018).

4 No se desarrolla extensivamente esta tesis, pero puede consultarse una amplia justificación al respecto en Esparza, 2019. Aclaramos este aspecto a pie de página porque consideramos que asignar a la educación y la formación como objetos formales de la pedagogía contiene un trasfondo teórico importante, pero que por ahora no podemos desarrollar. 
- Por comunidad de personas reconocemos no a los grupos de individuos sino a las distintas instituciones pedagógicas que actualmente conforman nuestra sociedad. Consideramos que la pedagogía debe intervenir para formar a la persona, pero reconociendo el marco institucional en el que ella se desarrolla. La aproximación a la realidad del ser antropológico debe asumir la realidad de la estructura social en la que se desenvuelve, de tal modo que se considere a la persona en su dimensión sustancial y en su dimensión cultural. Así, su formación está directamente vinculada a la forma de la institución social que la antecede.

De acuerdo con esto, podemos encontrar diversas estrategias dentro del estado de la cuestión que permiten intervenir y modificar conductas relacionadas con el consumo de alimentos. Presentamos algunas de las más significativas al respecto. Del Valle y Solleiro (2003) reconocen la importancia de las industrias en la alimentación en México, resaltando los siguientes retos:

a. La competitividad y agenda (menú) que imponen cadenas internacionales.

b. La rentabilidad de los productos consumidos en México (¿es posible alimentar sanamente $y$, al mismo tiempo, ser rentable?).

c. Los límites entre investigación en alimentos y prácticas económicas necesarias para el desarrollo de la industria (¿es posible conciliar la investigación y el consumo natural de alimentos?).

Como se apreciaba en el apartado final de la pregunta anterior, las industrias asumen tres retos reconocidos: competitividad de productos, rentabilidad e innovación. Lo interesante de estos retos apunta directamente a las acciones que se están impulsando tanto para fomentar hábitos -lo que ya comemos-, como también nuevos intereses de consumo -lo que se nos promueve a comer-.

La pregunta por los hábitos alimenticios está íntimamente ligada con las ofertas de mercado. Comemos lo que "podemos» comer. En ese sentido, y de acuerdo con la Encuesta Nacional de Salud y Nutrición (ENSAUT, 2018-2019), y a lo ya analizado, en México es posible la alimentación, aunque no necesariamente esta conlleva una sana alimentación. De acuerdo con la ENSAUT, no comemos dignamente: nuestro país enfrenta el problema del consumo y la obesidad. Quienes comemos, afrontamos el reto de alimentarnos «dignamente». En lo que respecta a los retos, el trabajo compilado por Del Valle y Solleiro (2003), así como la ENSAUT (2018-12019), apuntan a los siguientes:

- Mayor flexibilidad por parte de la industria. Se promueve el reconocer las necesidades alimentarias y enfocarse no solo en los retos de la rentabilidad, sino también de la nutrición. La pregunta a resolver es: ¿las empresas se preocupan seriamente por lo que comemos, tanto como se preocupan por cuánto venden?, ¿cuál es el objetivo real de las estrategias comerciales de nuestros productos?

- Mejorar la (in)formación dietética del consumidor. Desde las empresas, deben procurarse mejores prácticas alimenticias para el consumidor. Si bien el objetivo de una industria parece agotarse con señalar la información nutrimental y el cuidado de la calidad de los alimentos, el reto a resolver sería: ¿es suficiente con el etiquetado?, ¿qué otras acciones pueden promoverse para formar hábitos éticos y dietéticos responsables?, ¿es la empresa responsable de estas tareas?

A las preguntas se responde afirmando que el objetivo de una pedagogía del consumo es impulsar y educar para comer «saludablemente», y enseñar los estándares médicos y 
nutriológicos convenientes para el desarrollo de nuestro cuerpo. Entendemos que el reto más importante se encuentra en los procesos que impulsan las industrias de alimentos a través de sus programas y agendas (menú), promoviendo un cierto tipo de alimentación: no siempre lo ofertado es lo más saludable, sino lo más conveniente comercialmente.

De acuerdo al ENSAUT, en México sufrimos una crisis de obesidad y malnutrición derivada de nuestros malos hábitos como consumidores, y también como parte de la promoción de las industrias. Las industrias de alimentos, deben flexibilizar comercialmente sus estrategias de promoción de consumo. El reto, anotábamos ya, es: ¿cómo generar mejores ingresos sin atentar contra la salud alimentaria de los consumidores?, ¿cómo conciliar ambas esferas?

Uno de los desafíos más importantes en el desarrollo del programa de Negocios Gastronómicos, tiene que ver justamente con la concientización de los recursos pedagógicos y de formación de hábitos que debe tener en cuenta el empresario, para el desarrollo de nuevas visiones y estrategias de organización de los negocios. La finalidad del programa -además de las claramente administrativas y gastronómicas- es la concientización de los procesos requeridos para el desarrollo de habilidades que permitan a los actuales empresarios, y alumnos en general que cursan el programa, el considerar a la persona como el centro de la actividad de su negocio. Desde luego, no se pueden desprender del desarrollo de las acciones propias que promueve la industria de los alimentos (rentabilidad y legalidad), pero no por ello deben descuidarse aspectos esenciales de la estructura antropológica que compone a una empresa.

Sostenemos que toda actividad comercial debe impulsar necesariamente el desarrollo de la dignidad de la persona pero, dada la naturaleza de la actividad comercial, es vital impulsar hábitos de consumo responsables que ayuden a contrarrestar los excesos y las desinformaciones en la producción, distribución, publicidad y compra de alimentos.

El problema implica un reto multidisciplinar que la pedagogía debe afrontar. Un primer paso hacia la solución es visibilizar y estructurar los medios de promoción y patrones de consumo entre los consumidores. Se espera que este artículo ofrezca, pues, ese primer paso, en esta línea trascendental.

\section{CONCLUSIONES}

Se mostró que, de acuerdo al ENSAUT, en México sufrimos una crisis de obesidad y malnutrición derivada de nuestros malos hábitos como consumidores, de la falta de difusión de información nutricional confiable, y también de la promoción -por mucho tiempo no regulada- de las industrias.

En su dimensión comercial, las industrias de alimentos deben flexibilizar sus estrategias de promoción de consumo. El reto es: ¿cómo generar mejores ingresos sin atentar contra la salud alimentaria de los consumidores?, ¿cómo conciliar ambas esferas?

El etiquetado frontal de los alimentos en México -recientemente actualizado con la modificación a la NOM-051-, representa un avance en cuanto a una comunicación más transparente entre industria de alimentos y consumidores; sin embargo, una única estrategia no es suficiente para tratar todos los problemas de salud relacionados con el comportamiento del 
consumo de alimentos: se evidencia la necesidad de que la industria retome como prioridad el producir alimentos nutritivos y adecuados para sus consumidores, dejando la publicidad en un plano secundario, $y$, en un escenario ideal, reinventando esa publicidad como una forma de educar a los consumidores, fomentando que desarrollen la capacidad de tomar mejores decisiones sobre su alimentación.

Esta modificación a la NOM-051 ciertamente ha despertado un interés más profundo de la industria por cuidar su imagen, su calidad y, por supuesto, a sus consumidores. Una gran cantidad de alimentos se han sometido a reformulación, eliminación o sustitución de ingredientes de bajo aporte nutricional; y en algunos casos importantes, dentro de esa reinvención, se ha considerado la importancia de la pedagogía del consumidor: casos en los que los alimentos incluyen recomendaciones, leyendas de salud, direccionamiento a páginas web con mayor información, uso de códigos $(\mathrm{QR})$ para acceder a más información sobre el alimento o la familia de alimentos a la que pertenece el producto.

En una primera fase, la modificación de la norma se centra en indicar a los consumidores, los elementos críticos en los alimentos, mostrando información clara que protege contra publicidad engañosa (Comité de expertos académicos nacionales del etiquetado frontal de alimentos y bebidas no alcohólicas para una mejor salud, 2018).

Esperaríamos que en etapas posteriores, la comunicación entre los productores de alimentos y consumidores, a través del etiquetado, evolucione en el sentido de que la población pase de estar informada (formada) a aplicar esa información para tomar mejores decisiones de acuerdo con sus gustos y necesidades, o restricciones nutricionales (educación). En este sentido, el etiquetado pudiera empezar a enfocarse también en ingredientes benéficos para la salud y no solo en alertar a los consumidores sobre aquellos que representan un riesgo.

La regulación gubernamental debe ser parte, de manera congruente, de las estrategias que permitan un acercamiento más objetivo del consumidor con sus alimentos. La modificación del etiquetado frontal debe acompañarse de campañas en las que se provea de educación nutricional, de manera que se den las condiciones adecuadas para que los consumidores comprendan sus necesidades nutricionales y, asimismo, tengan las herramientas para reflexionar sobre los mejores alimentos y, así, cubrirlas.

De acuerdo con las conductas actuales de consumo de alimentos en México -posicionado como uno de los factores diferenciadores el NSE-, se evidencia un área desatendida por las autoridades de salud y la misma industria alimentaria. La distribución y el aseguramiento de alimentos saludables y adecuados para toda la población, debería ir de la mano con la forma de cubrir requerimientos nutricionales de cada individuo, sin que la calidad ni la funcionalidad se vieran comprometidas. El fomento de nuevas conductas hacia los alimentos puede basarse en los productos locales que sean asequibles para las poblaciones, sin que sea primordial el posicionamiento de alimentos industrializados de menor calidad nutricional. 


\section{REFERENCIAS}

Anastasiou, K.; Miller, M. \& Dickinson, K. (2019). The relationship between food label use and dietary intake in adults: A systematic Review. Appetite, vol. 138, 280-291.

Ayala Ramírez, Suhey y Castillo Girón, Víctor (2014). La distribución de alimentos y bebidas en México: una perspectiva desde el comercio tradicional. Espacio Abierto, vol. 23, no. 4, 661-681.

Calabrese, Claudio. (2018). Comunidad y Pedagogía. La Filosofía platónica de la educación. Zacatecas: Texere.

Carrete, Lorena; Arroyo, Pilar y Centeno, Edgar (2018). ¿Es la extensión de marca para productos saludables una estrategia adecuada cuando hay una alta asociación de la marca con productos no saludables? Revista Brasileira de Gestão e Negócios, vol. 20, no. 3, 421-442.

Castronuovo, Luciana; Gutkowski, Patricia; Tiscornia, Victoria y Allemandi, Lorena (2016). Las madres y la publicidad de alimentos dirigida a niños y niñas: percepciones y experiencias. Salud Colectiva, vol. 12, no. 4, 537-550.

Comenio, Juan Amos (1992). Pampedia. (Educación Universal) Estudio Preliminar y Traducción de Federico Gómez R. de Castro. Madrid: UNED.

Kaufer-Horwitz, M.; Tolentino-Mayo, L.; Jáuregui A. et. al. (2018). Sistema de etiquetado frontal de alimentos y bebidas para México: una estrategia para la toma de decisiones saludables. Salud pública Mex, vol. 60, no. 4, 479-486.

Del Valle, Carmen; Solleiro, José (2003). Estrategias competitivas de la Industria Alimentaria. México: Plaza y Valdéz.

Denham, D. \& Gladstone, F. (2020). Making sense of food system transformation in Mexico. Geoforum, 115, 67-80.

ENSAUT (2019). Encuesta Nacional de Salud 2018-2019. México: Instituto Nacional de Salud Pública. https: / / ensanut.insp.mx/

Esparza, Gustavo, coord. (2019). Pedagogía y Ética. Dilemas didácticos de la formación profesional. Zacatecas: Texere.

Galván-Portillo, M.; Sánchez, E.; Cárdenas-Cárdenas L. M. et al. (2018). Dietary patterns in Mexican children and adolescents: Characterization and relation with socio-economic and home environment factors. Appetite, 121, 275-284.

Gaona-Pineda, E. B.; Martínez-Tapia, B.; Arango-Angarita, A.; Valenzuela-Bravo, D.; GómezAcosta, L. M.; Shamah-Levy, T. y Rodríguez-Ramírez, S. (2018). Consumo de grupos de alimentos y factores sociodemográficos en población mexicana. Salud Pública de México, vol. 60 , no. $3,272-278$.

Gil-Toro, Daniela; Giraldo-Giraldo; Nubia A. y Estrada-Restrepo, Alejandro (2017). Ingesta de alimentos y su relación con factores socioeconómicos en un grupo de adultos mayores. Salud Pública de México, vol. 19, no. 3, 304-310. https:/ / doi.org/10.15446/rsap. $\underline{\mathrm{v} 19 \mathrm{n} 3.54804}$ 
Hernández-F, M.; Figueroa, J. L. y Colchero, M. A. (2021). Association between density of stores and purchases of ultra-processed food and sugar-sweetened beverages in Mexico. Health and Place, no. 68, 102528.

Ibarrola-Rivas, M. J. y Galicia, L. (2017). Rethinking Food Security in Mexico: Discussing the Need for Sustainable Transversal Policies Linking Food Production and Food Consumption. Investigaciones Geográficas, IG, no. 94. UNAM.

Jansen, E. C.; Marcovitch, H.; Wolfson, J. A.; Leighton, M.; Peterson, K. E.; Téllez-Rojo, M. M.; Cantoral, A. \& Roberts, E. F. S. (2020). Exploring dietary patterns in Mexican adolescent population: A mixed methods approach. Appetite. 147, 104542. https://doi. org/10.1016/j.appet.2019.10454

Junco, Ethel (2019). La Apología de Sócrates como texto preliminar para la Ética de las Profesiones. En Esparza, Gustavo, coord., Pedagogía y Ética. Dilemas didácticos de la formación profesional, 35-58. Zacatecas: Texere.

Llergo, Fernanda y Nicolás, Teresa (coords). (2017). La filosofía educativa de la Universidad Panamericana. Excelencia Académica. Excelencia Humana. México: Universidad Panamericana.

López Ojeda, Andrés; Pérez Camacho, Carmen; Guzmán Hernández, Celia y Hernández López, Ricardo (2017). Caracterización de las principales tendencias de la gastronomía mexicana en el marco de nuevos escenarios sociales, Teoría y Praxis, no. 21, 91-113.

Luria, A.; Leontiev, A. y Vigotsky, L., (2018). Psicología y Pedagogía. Edición castellana de Esther Benitez Akal Bolsillo: Madrid.

MacFie, H. \& Thomson, D. (eds.) (1994). Measurement of Food Preferences, London: Springer.

Marrón-Ponce, Joaquín; Fernández-Gaxiola, Ana; Cruz-Casarrubias, Carlos; García-Guerra, Armando; Pacheco-Miranda, Selene; Quezada, Amado; Pérez-Luna, Miriam y Donovan, Jason (2021). Perfil nutricional y estrategias de publicidad en el empaque de alimentos procesados de trigo y maíz en la Ciudad de México. Salud Pública de México, no. 63, vol. $1,79-91$.

Moreno y de los Arcos, Enrique (1993). Principios de Pedagogía asistemática. Ensayos. México: UNAM.

Moskowitz, H. R. (1994). Product optimization: approaches and applications. In MacFie, H. \& Thomson, D. (eds.) (1994). Measurement of Food Preferences, London: Springer.

Moss, Michael (2013). Salt, Sugar, Fat. How the Food Giants Hooked Us. New York: Random House.

NOM-051-SCFI/SSA1-2010. DOF. Viernes 27 de marzo 2020.

Nout, Luis (2017). Pedagogías del conocimiento. México: Fondo de Cultura Económica.

Pérez-Salgado, Diana; Rivera-Márquez, José y Ortiz-Hernández, Luis (2010). Publicidad de alimentos en la programación de la televisión mexicana: ¿los niños están más expuestos? Salud Pública de México, vol. 52, no. 2, 119-126.

Suárez-Solana, M. Carmen (2016). Significado externo de «alimentación correcta» en México. Salud Colectiva, vol. 12, no. 4, 575-588. 
Sunstein, C.R. (2021). Viewpoint: Are food labels good? Food Policy, 99, 101984.

Taddei Bringas, Cristina. (2006). Estrategias de mercado en firmas líderes de la industria alimentaria. Estudios Sociales, vol. 15, no. 28, 68-106.

Tolentino-Mayo, L.; Sagaceta-Mejía, J.; Cruz-Casarrubias, C.; Ríos-Cortázar, V.; Jauregui, A. y Barquera, S. (2020). Comprensión y uso del etiquetado frontal nutrimental. Guías Diarias de Alimentación de alimentos y bebidas industrializados en México. Salud Pública de México, no. 62, vol. 6, 786-797.

Tolentino-Mayo, Lizbeth; Rincón-Gallardo, Sofía; Bahena-Espina, Liliana; Ríos, Víctor y Barquera, Simón (2018). Conocimiento y uso del etiquetado nutrimental de alimentos y bebidas industrializados en México. Salud pública de México, vol. 60, no.3, 328-336. https://doi.org/10.21149/8825

Vique-Sánchez, José Luis y Galindo-Hernández, Octavio (2021). México con alta prevalencia de enfermedades crónico-degenerativas y factores de riesgo que favorecen desarrollar COVID-19. Nutr. Clín. Diet. Hosp., 41(1), 55-60. https:// doi.org/10.12873/411vique 